Cahiers $d u$ MONDE RUSSE

\section{Cahiers du monde russe}

Russie - Empire russe - Union soviétique et États indépendants

$60 / 4 \mid 2019$

Varia

\title{
Manfred ZELLER, Sport and Society in the Soviet Union. The Politics of Football after Stalin
}

\section{Sylvain Dufraisse}

\section{OpenEdition}

\section{Journals}

Édition électronique

URL : https://journals.openedition.org/monderusse/11532

DOI : $10.4000 /$ monderusse. 11532

ISSN : $1777-5388$

Éditeur

Éditions de l'EHESS

\section{Édition imprimée}

Date de publication : 1 octobre 2019

Pagination : 861-864

ISBN : 978-2-7132-2797-4

ISSN : 1252-6576

Référence électronique

Sylvain Dufraisse, "Manfred ZELLER, Sport and Society in the Soviet Union. The Politics of Football after Stalin », Cahiers du monde russe [En ligne], 60/4 | 2019, mis en ligne le 01 octobre 2019, consulté le 05 janvier 2023. URL : http://journals.openedition.org/monderusse/11532 ; DOI : https://doi.org/ 10.4000/monderusse.11532 
Manfred ZELLER

\section{Sport and Society in the Soviet Union}

The Politics of Football after Stalin

Londres - New-York : J.B. Tauris, 2018, 300 p.

Le 20 octobre 1982, à l'issue d'un match de coupe UEFA entre le Spartak de Moscou et l'équipe néerlandaise HFC Haarlem, un mouvement de foule dans le stade Lužniki de Moscou provoque la mort de soixante-sept personnes, auxquelles s'ajoutent soixante-et-un blessés, pour la plupart de jeunes fans. Les causes de cette tragédie, comme le nombre de victimes, ont été l'objet de controverses. D'aucuns ont accusé les responsables soviétiques de minimiser cette catastrophe et les responsabilités des services chargés de la sécurité du stade. Manfred Zeller part de cet épisode, dont il compare la mémoire avec la victoire contre l'équipe allemande détentrice de la coupe du monde en 1955 et l'envahissement du terrain par les supporters du Dinamo de Kiev en 1956 à Moscou, pour faire surgir les liens et les clivages qui traversèrent cette passion «ordinaire» (C. Bromberger) : les matchs de football.

Cet ouvrage, traduit de la version allemande parue en 2015, étudie comme le titre dans sa version originale l'indiquait la «fièvre du football» dans l'URSS poststalinienne. Il s'inscrit ouvertement dans la lignée des travaux de Robert Edelman sur le spectacle sportif et le développement du football en Union soviétique ${ }^{1}$, qu'il complète et nuance, et dans la riche école allemande d'analyse des pratiques corporelles et sportives en Russie impériale et en Union soviétique que Nikolaus Katzer a fédérée ${ }^{2}$. Il intéressera bien évidemment les historiens et sociologues spécialistes du sport, tant il apporte sur l'émergence du supportérisme à l'Est et sur les formes soviétiques de soutien aux équipes, mais il doit aussi susciter l'intérêt vif des historiens de la société soviétique. En effet, Manfred Zeller étudie la diffusion du goût pour le match de football, à la radio, à la télévision et dans les stades en URSS. Il l'inscrit dans les transformations sociales, politiques et culturelles qui ont touché la société soviétique depuis la mort de Stalin. Comme l'auteur le rappelle, il n'y a pas eu en URSS de pénurie de matchs de football! Ce faisant, il concourt à éclairer le phénomène de démocratisation et d'appropriation des loisirs en Union soviétique et il démontre comment, dans la période soviétique, le spectacle sportif a contribué à «faire» des Soviétiques. Par le prisme de la «fièvre du football», Manfred Zeller réalise de manière convaincante une étude de la «société participative $^{3}$ » et montre comment le football a permis en Union soviétique l'identification à des communautés (nationales, de clubs, de villes), sans forcément que cela dépende 
uniquement du lieu de résidence ou de la nationalité d'origine, et l'attachement à la «Grande Patrie».

Dans ce but, l'auteur a constitué un large corpus de sources inédites tirées des archives centrales politiques, administratives et sportives. Afin de décentrer le regard et d'éclairer aussi le rapport à l'un des fleurons du football soviétique, le Dinamo de Kiev, premier club soviétique à remporter des titres européens en 1975, Manfred Zeller a dépouillé de nombreuses archives ukrainiennes. Ainsi, il souhaitait comparer les formes d'attachement aux équipes et saisir en quoi le spectacle sportif avait pu favoriser la manifestation de nationalismes. À cet ensemble, déjà très fourni, s'ajoutent également les fonds de la ville de Moscou et des fonds iconographiques (TsDKU, Fotoagenstvo Sport-Ekspress de Moscou), le dépouillement de la presse sportive et des entretiens avec des journalistes sportifs, d'ex-Soviétiques amateurs de football et des supporters. Cet ensemble dense de matériaux lui a permis de dépasser la mythologie aujourd'hui constituée par les clubs ou les groupes de supporters dominants, de saisir les processus de diffusion et de constitution d'un espace commun de pratique, de revenir sur la pluralité des mémoires, qui dépasse le seul soutien au Spartak «équipe du peuple» contre le Dinamo, équipe du NKVD.

Le premier chapitre, historiographique, participe à la définition de l'objet de recherche : la «fièvre du football». L'auteur inscrit la consommation et la réception de matchs de football dans le cadre de la culture populaire poststalinienne. Il met alors en avant un des objectifs de son projet : étudier comment le goût du football permet de façonner une communauté soviétique. Le deuxième chapitre revient sur la genèse du phénomène du supportérisme et exhume les premières traces concrètes en URSS. Le football touche principalement les villes dans les années 1920 et 1930. L'accès au spectacle sportif est limité : le coût d'un billet pour un match est élevé. Seules les grandes villes disposent de stades et d'équipes de football de renom. L'ensemble du territoire soviétique n'a pas accès aux dispositifs de radiodiffusion. Le développement et la diffusion des radios et de la télévision contribuent à élargir le phénomène dans la période d'après-guerre, à toucher une audience plus large et à créer des communautés de fans, car la consommation des matchs autour du poste est une expérience collective. Manfred Zeller reconstruit l'attachement aux équipes. L'auteur montre qu'en parallèle à la couverture médiatique plus importante des matchs de football naissent et croissent des récits fondateurs et des communautés mémorielles autour des équipes. Il bouscule un mythe bien ancré chez les chroniqueurs footballistiques, le Spartak comme «équipe du peuple» et en opposition au régime. Le soutien au Spartak, équipe très populaire dans les années 1930, s'explique par son jeu différent et plus offensif, par l'importance que ses dirigeants ont donnée au divertissement sportif, mais aussi par la capacité des Starostin à manœuvrer au sommet du régime soviétique et à se protéger par le biais de patronages puissants. Le soutien au Dinamo et au TsDKA est plus tardif. Il se développe dans les années 1940, parallèlement aux tournées et aux succès de ces équipes. Il s'organise autour d'un récit mythique, liant les victoires footballistiques aux institutions dont ils dépendent - l'armée et les services de sécurité - et aux hauts faits guerriers durant la Seconde Guerre mondiale. Quant à l'équipe d'URSS, 
si les matchs contre les Basques en 1937 ont rassemblé le public soviétique amateur de football, c'est véritablement le 21 août 1955 que l'attachement à l'équipe nationale s'affirme. La victoire contre l'équipe de RFA est perçue comme un second Stalingrad. Elle fait communier les générations, les familles et les supporters des différentes équipes moscovites.

La télévision est un des canaux privilégiés de la diffusion du goût du football. Alors que le temps disponible augmente, la télévision devient un des loisirs principaux des Soviétiques au cours des années 1960-1970. Le sport y tient une place importante. La consommation de matchs est collective, transgénérationnelle, mais elle est surtout masculine. Les téléspectateurs, parfois indignés, s'adressent à la télévision et à la radio soviétiques pour dénoncer des décisions partiales de l'arbitre ou les remarques des commentateurs. Certains se retrouvent dans des lieux de discussions informels pour échanger sur leurs équipes préférées. Les citoyens soviétiques, devant les matchs de football, imaginent l'Union soviétique comme un espace d'oppositions internes. Ils étoffent également leur connaissance du football et des clubs engagés dans le championnat et partagent des expériences.

La «fièvre du football» atteint aussi les tribunes des stades. Manfred Zeller, contre l'image de stades pacifiques, revient à partir des rapports d'arbitres, de police et d'articles de presse, sur les manifestations de violence et de mécontentement présentes dès les années 1950. Insultes à l'égard des joueurs et des arbitres, jets d'objets, envahissement de terrains, émeutes brutales; la gamme des manifestations hostiles est variée et s'articule autour de deux principales revendications : des erreurs d'arbitrage et des oppositions nationales. À partir de la fin des années 1950, le traitement de ces incivilités passe par une police des comportements : contrôle plus fin des enceintes sportives, censure de la presse et de la télévision, régulation de l'entrée des stades et «éducation» des supporters. Les tribunes des stades sont aussi l'occasion de transferts culturels. Les retransmissions des championnats d'Europe de l'Ouest et d'Amérique latine contribuent à populariser de nouvelles formes d'engagements par le port des couleurs du club ou des drapeaux. Dans le contexte de pénurie soviétique, les grand-mères ou les mères, voire les supporters eux-mêmes, tricotent des écharpes aux couleurs de leur club favori. D'autres personnalisent leurs foulards de pionniers, parfois même des drapeaux de leur section du Komsomol. Dépassant la vision de groupes violents apparus durant la perestroïka et visibles dans les années 1990, venus de l'Ouest, il montre comment les groupes de supporters se fédèrent au cours des années 1970 par des expériences communes (les voyages réguliers à l'étranger, le suivi des équipes aux confins de l'URSS, l'interconnaissance des groupes de supporters des clubs moscovites...) et comment ils se hiérarchisent par leurs manifestations dans les stades. Si cette pratique est d'abord moscovite et plutôt bien acceptée par les services de sécurité, les luttes de territoire et les affrontements qui éclatent entre fans poussent les autorités à surveiller de manière bien plus attentive ce qui devient une culture alternative. Malgré les tentatives d'encadrement par le Komsomol, les mouvements de supporters qui rassemblent une population plus âgée et plus homogène, s'autonomisent et se divisent durant les années 1990 entre ultras dans les stades et hulsy, 
spécialisés dans les oppositions violentes. Ce mouvement n'est bien sûr pas uniquement russe ; il s'inscrit dans une tendance européenne de montée des violences dans et autour des stades.

Enfin, et c'est un des points importants du livre, si la télévision et le football avaient produit des spectacles rassemblant la communauté soviétique, le soutien au club montre une gamme des appartenances au sein même de l'Union. En étudiant le soutien au Dinamo de Kiev, équipe phare des années 1970 et 1980, Manfred Zeller complète l'étude du «supportérisme à distance ${ }^{4} »$. Lorsque l'équipe de la RSS d'Ukraine jouait à l'étranger, l'ensemble des fans de football était rassemblé derrière eux. Le football permet de «vivre le socialisme». Des lettres de soutien arrivent de tout le territoire : cette équipe symbolise l'amitié entre les peuples. Dans le cadre du championnat national, certains fans des républiques socialistes soviétiques se fédèrent autour d'une équipe «périphérique» défiant le centre moscovite; d'autres enfin vivent au travers des couleurs de cette équipe l'attachement à la «petite patrie» originelle, l'Ukraine. En RSS d'Ukraine, Manfred Zeller retrace à partir des lettres reçues un attachement différencié : les fans du Dinamo résident davantage dans l'Ouest et l'Ukraine centrale.

Manfred Zeller s'est donc attaché par ce livre à porter un autre regard sur la période khrouchtchévienne et brejnévienne. Malgré les souvenirs douloureux du passé, une partie de la population a pris part, par le biais du football, à une communauté soviétique avec ses chroniques, ses rivalités internes et ses derbys qui ont contribué à donner du sens à un tout, l'URSS. Mais il montre aussi que c'est le renforcement du contrôle, sensible au tournant des années 1980, à l'égard des mouvements de jeunesse et les formes de police à l'œuvre qui concourent à faire de la culture des supporters de football une culture informelle et alternative, ferment de la glorification nationaliste et de la perpétration d'actions xénophobes au début des années 1990. L'ouvrage de Manfred Zeller témoigne de l'intérêt d'utiliser le sport comme un analyseur et illustre combien l'étude de cet aspect de la vie sociale, parfois déconsidéré, permet de saisir par d'autres biais la construction de la société soviétique, au quotidien.

1 - Robert Edelman, Spartak Moscow : A History of the People's Team in the Worker's State, Ithaca - Londres : Cornell University Press, 2009 ; Robert Edelman, Serious Fun : a History of Spectator Sports in the USSR, Oxford : Oxford University Press, 1993.

2 - Nikolaus Katzer et alii, éds., Euphoria and Exhaustion, Modern Sport in Soviet Culture and Society, Frankfurt - New York : Campus Verlag, 2010.

3 - Klaus Gestwa, « Reflektierte Archivarbeit - der "Königsweg” osteuropäischer Zeitgeschichte. Die übersichtliche «Welt der Modelle» und die «konstitutive Widersprüchlichkeit» des Sowjetsystems », OstEuropa, 50, 5 (2003), p. 554.

4 - Ludovic Lestrelin, L'autre public des matchs de football : Sociologie des supporters à distance de l'Olympique de Marseille, P. : EHESS, (« En temps et lieux »), 2010.

\section{Sylvain Dufraisse}

Centre de Recherches en Histoire des Slaves, Institut Pierre Renouvin, Université Paris 1 Panthéon-Sorbonne. 